\title{
Effects of beam focusing on the efficiency of planar
} waveguide grating couplers

\author{
Lifeng Li and Mool C. Gupta
}
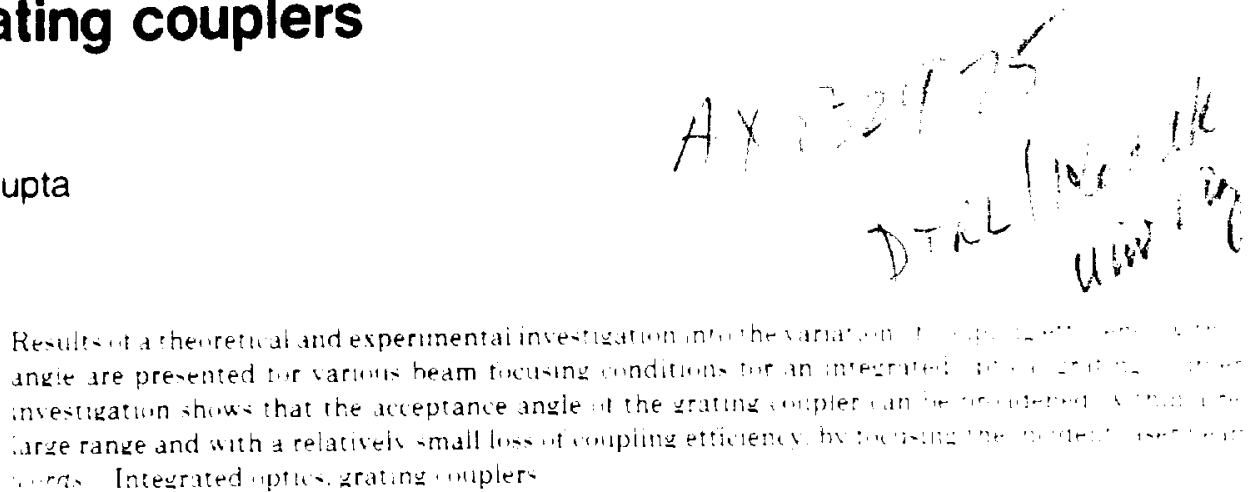

\section{Introduction}

Diffraction gratings play an important role in integrated optics: they are used to couple light into waveguides; to focus. deflect. and split beams: and to tilter light spectrally tas in distributed feedback lasers Grating couplers are not only durable, they can be fabricated through planar photolithographic techniques.

A distinctive feature of a periodic grating coupler is that its coupling efficiency is high only if the incident beam falls within a narrow range of coupling angles." In many cases this feature can be used to some advantage. For some applications, however, it is desirable to render the coupling efficiency less sensitive to the coupling angle. One way to achieve this is by focusing the incident beam. A focused beam can be considered a bundle of rays with a range of angular components. When this angular range covers the optimum coupling angle. a portion of the bundle will be coupled into the waveguide and the remainder will not. Thus, the curve of coupling efficiency vs coupling angle will be broadened, but its peak height will be lowered.

Even though grating couplers are widely used in integrated optics, and even though the theory of grating couplers is well understood, to our knowledge little detailed study has been done on the angular dependence of the coupling efficiency for focused beams. In

Lifeng $\mathrm{Li}$ is with Iniversity of Arizona. Optical Sciences Center. Tucson. Arizona 8.5:21. and Mool Gupta is with Eastman Kodak Cumpany. Research Laboratories. Rochester. New York 14650 . 2017 .

Received 20 November 1989

(j00)3-5935/90/365.320-1)6\$02.00/0.

c 1990 Optical Society of America. this paper we report the results of uur theuretical and experimental investigation into the variation with coupling angle of the coupling etficiency tor an integrated optical grating coupler. Various tocused beam condi. tions were explored in our investigation. The impur. tant question we address is how great an angular broadening can be achieved for a given luss ot peak efficiency.

\section{Theory}

The analysis of a grating coupler requires two calculations: (1) the calculation of the effective index and leakage factor of the corrugated waveguide. and $(2)$ the calculation of the coupling efficiency. For the first calculation we use Maystre's integral method." For the second calculation we use the method developed by Neviere et al. ${ }^{j-3}$ This method consists of three steps. First, an incident beam of finite width is represented by a superposition of plane waves. Second. the response of the grating coupler for a plane wave in the vicinity of the waveguide resonance is represented by a complex pole. Third, the total response of the coupler to the finite beam is given by the superposition of its response to plane waves. Neviere et al. ${ }^{-}$considered only the case in which the incident angle is phase matched with the grating coupler, and the incident beam has no phase variation other than that of a plane wavefront. In our work we considered the general case. By allowing an arbitrary incident angle and arbitrary phase variation, we studied the angular dependence and defocus dependence of the device 's coupling characteristics. Throughout this paper we assume that $l \gg 1 / \alpha^{\prime}$, where $l$ is the grating length and $\alpha$ is the leakage factor defined below. This condition is well satisfied by the grating couplers used in our ex. periment.

Following the analysis in Refs. 7 and 8 . one can 
derive the fillowing expression for the coupling etficiency tor a lissless. intinitely long grating coupler:

$\cdots+\ldots+\cdots h-\cdots$

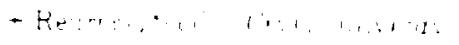

where $r$ is the poition along the grating and $t$ is measured along the nurmal to the waveguide: $k=2 \pi$ 1. With the watiensth in a racuum: $n$ is the retractive index of the inciuent medium: $C(\cdots)=1$ for $T E$. and $(i)=1$ tor TM. with 6 the dielectric constant and $C$

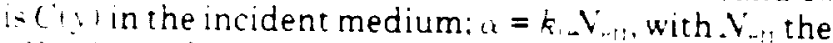
effective index of the guided mode: $K=2 \pi$. with $d$ the grating period: $m$ is the diffraction order which gets coupled into the wareguide: $\iota_{n}$, is the $m$ th order field amplitude excited by an incident plane wave on resonance: and $q(x)$ gives the incident beam profile. normalized such that $f y-d t=1$, with $t$ perpendicular to the beam axis.

The variables detined above are properties of the grating coupler and of the incident beam. The interaction between the coupler and the incident beam is described completely by the function $r(x)$, which can be stated in three equivalent forms:

$$
\begin{aligned}
& \frac{d r|x|}{d x}+14 x-n|r| x|=q| x \mid . \\
& -(x)=-\varepsilon \quad 01 \xi) \frac{1}{\xi+\left(x-\alpha_{3}\right.} \exp (i \xi x) d \xi . \\
& r \mid x:=f q(x) H\left(x-x^{\prime} \mid \exp \left[a 1 \alpha-(x) \mid 1 x-x^{\prime}\right)\right] d x^{\prime} .
\end{aligned}
$$

where $p(\xi)$ is the Fourier transform of $q(x) ; \alpha_{1\}}=n k_{1}$ $\sin \theta_{11}$ is the center spatial frequency of $p(\xi) ; \theta_{11}$ is the incident angle: $\alpha_{0}=n k_{1} \sin \theta_{R}+i \alpha^{\prime}$ is the complex pole of the waveguide grating structure; $\theta_{R}$ is the resonance coupling angle: $\alpha^{\prime}$ is the leakage factor; and $H\left(x-x^{\prime}\right)=$ 1 if $x-x^{\prime}>0$ and $H\left(x-x^{\prime}\right)=0$ if $x-x^{\prime}<0$. Equation (2) is probably the most useful for a numerical computation of $r(x)$, but Eqs. (3) and (4) are more useful for an intuitive understanding of the dependence of $r(x)$ on the incident angle or beam size (angular width) of the incident beam. We define several terms to simpliiy our discussion. $\theta \equiv \theta_{1}-\theta_{R}$, the angular detuning. We define the full width at half maximum of the $\eta$ vs $\theta$ curve, the apparent acceptance angle (or, simply, the acceptance angle) of the grating coupler, and denote this by $\Delta \theta_{\text {. }}$. Clearly, $\Delta \theta_{a}$ depends both on the grating coupler and on the incident beam. It can be shown ${ }^{3}$ that as the incident beam becomes a plane wave, $\Delta \theta_{a}$ tends to its lowest value $\Delta \theta_{i}=2 \operatorname{Im}\left[\alpha_{p}\right] /\left(n k_{n} \cos \theta_{R}\right)$, which is the full width at half-maximum of the Lorentzian profile $1:\left(\xi+\alpha_{11}-\alpha_{p}\right) \mid:$. We call $\Delta \theta_{1}$ the intrinsic acceptance angle of the grating coupler.

The angular dependence of $\eta(x)$ is contained in the function $r(x)$. When $\theta=0, r(x)$ as given by $\mathrm{Eq}$. (4) is a convolution of the incident beam profile and an exponential decay function. When $\theta \neq 0$, an oscillatory function is superimposed on the exponential decay function: therefore, the coupling efficiency decreases. In Eq. (3), $r(x)$ is given by an overlapping integral of

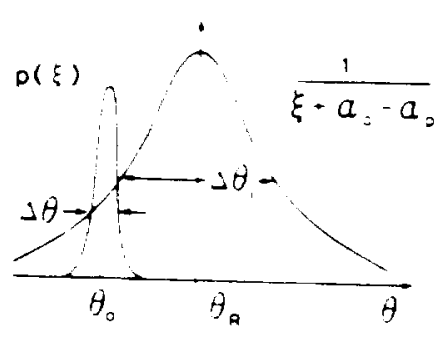

(a)

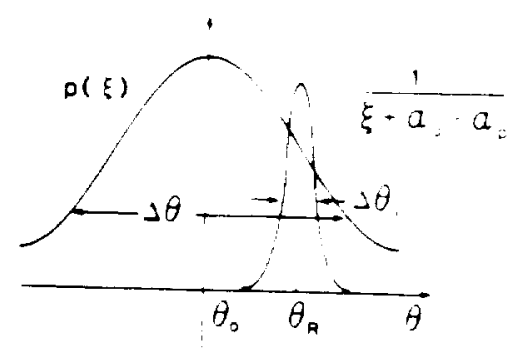

(b)

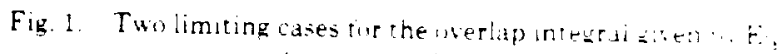

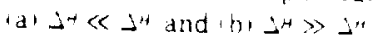

$p(\xi)$ and $1 /\left(\xi+\alpha_{17}-\alpha_{0}\right) \exp (i \xi x)$. When $\theta=0$, the peaks of the two functions coincide, and the maximum coupling efficiency is achieved. A nonzero detunins implies a shift in the two peaks and a decrease in? efficiency. When $\theta$ changes, the two curves mose relative to each other. If the angular width of incident beam $\Delta \theta$ is $\ll \Delta \theta$., the Lorentzian is probed by the incident beam: if $\Delta \theta$ is $\gg \Delta \theta$. the incident beam is probed by the Lorentzian (Fig. 1). In other words. the apparent acceptance angle approaches the intrinsic acceptance angle as $\Delta \theta$ tends to zero, and it approaches $\Delta \theta$ as the latter becomes $\gg \Delta \theta_{i}$.

Now let us consider a Gaussian beam, as depicted in Fig. 2. Once the wavelength and polarization are known. a Gaussian beam is specified completely by its beam waist $\omega_{1}$ or its angular width $\Delta \theta$. These $\left[\omega_{(}\right)$ quantities are related by $\lrcorner \theta=\tan ^{-1}\left(\lambda / \pi \omega_{1}\right)$. For our purposes it is more convenient to use the angular width. To specify the position of a Gaussian beam relative to a grating coupler we need three parameters: incident angle $\theta_{1,}$, lateral offset $x_{1,}$, and detocus $z_{1:}$. The lateral offset is defined as the distance between the beam axis and the edge of the grating. The defocus is defined as the distance between the beam's center on the grating to the beam waist, as shown in Fig. 2. We consider only the angular dependence, and we set $z_{1}=$ 0 . We consider the influence of defocus on the coupling efficiency at a later time.

\section{Exporimental Procedures}

The optical waveguide was prepared by e-beam de. position ${ }^{9.10}$ of a Corning 7059 glass film onto a quartz substrate with refractive index 1.46 . The thin tilm 


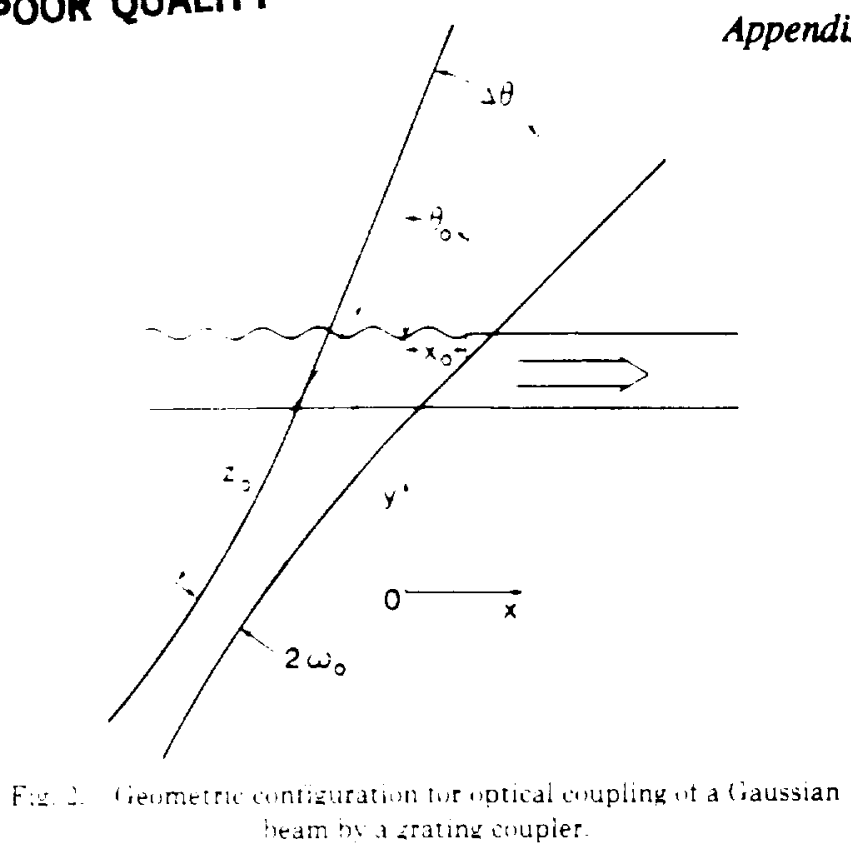

was $0.6 \mu \mathrm{m}$ thick. its refractive index was 1.50 , and its optical loss was $1 \mathrm{~dB} / \mathrm{cm}$. The waveguide cover is air. For TE polarization this waveguide supports only the lowest order guided mode. A surface relief grating was etched into the film at the air-film intertace. The grating was fabricated by the holographic technique. A $0.2 \cdot 2-u \mathrm{~m}$ thick photoresist (Shipley 1400 series) thin film was spin coated onto the quartz substrate. The resist was exposed with interference fringes $0.38 \mu \mathrm{m}$ apart ( using a $\mathrm{He}-\mathrm{Cd}$ laser with a wavelength of 0.4416 $\mu \mathrm{m})$. The exposed grating pattern was then developed. with an in situ monitoring technique. ${ }^{11}$ The relief pattern was transferred to the 7059 glass wavesuide by reactive ion beam etching with a $\mathrm{C}_{2} F_{n}$ gas. The ion beam etch rate for the film was found to be 15 $\mathrm{nm} / \mathrm{min}$ at an ion beam current density of $1 \mathrm{~mA} / \mathrm{cm}^{2}$. The shape of the grating rulings was rectangular. From a comparison of the measured and theoretically calculated diffraction efficiencies at several incident angles, we estimated that the grating groove had a depth of $0.1-\mu \mathrm{m}$. Two gratings, each $5 \mathrm{~mm}$ long, were etched $\sim 2 \mathrm{~cm}$ apart on a single sample, one grating to be used as the input coupler and the other as the output coupler.

The angular measurements were taken by mounting the grating coupler on a rotation stage with a $0.001^{\circ}$ resolution. Intensities of the transmitted, reflected, and throughput beams were measured with silicon detectors. The detector for the reflected signal had a large receiving area. so that the reflected beam would remain on the detector during the entire measurement process. The coupling efficiency measurements were performed with a polarized He-Ne laser with a Gaussian beam profile. The laser beam was expanded and nearly collimated, so that its angular halfwidth was $0.0085^{\circ}$, corresponding to a $2.7 \mathrm{~mm}$ diam beam waist. To obtain other angular widths, convex lenses of vari.
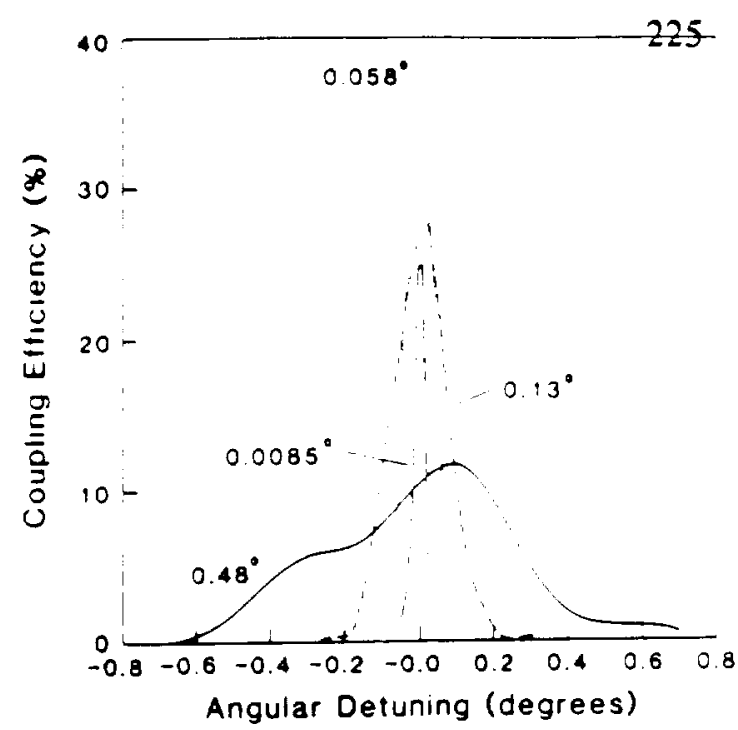

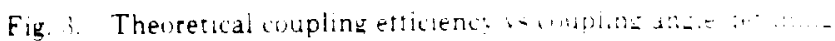

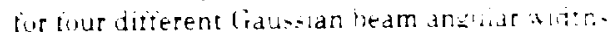

ous focal lengths were inserted after the beam expander. For all the measurements reported here. the in. put grating coupler was positioned at the beam wist: and the laser beam was TE polarized with respect : the grating coupler.

Each focused beam experiment proceeded as 6.1 lows: First, the optimum coupling condition was located iteratively by adjusting the lateral of fet and the coupling angle. so that the sum of the transmitted and reflected intensities was minimized. Once the ipt:mum condition was obtained. the lateral offset was fixed for the remainder of the measurement. and the coupling angle was referred to as the zero angular detuning. At the beginning of the measurement sequence, the grating was rotated to a negative detuning value at which the coupling efficiency was sufficienty. low. The grating was then rotated. in small increments, in the positive direction until the coupling efficiency was sufficiently low at a positive detuning value. The transmitted and reflected light intensities were measured at each angular position, from which the coupling efficiency was calculated.

\section{Results}

In Fig. 3, four theoretical curves for coupling efticiency vs angular detuning are plotted. For each curve the angular width of the incident beam is indicated.

Experimental data for variations in the coupling efficiency with coupling angle for four focused beam conditions are shown in Figs. 4- $i$. The percentage transmission, reflection, and throughput data were obtained. The throughput, that is, the light outcoupled by the second grating, is given in arbitrary units but is directly proportional to the coupling efficiency. The experimental coupling efficiencies were calculated by subtracting the percentage transmission and reflection from one hundred. We expect these coupling efficiency values to be somewhat higher than the actual values. because absorption and scattering are not taken into 

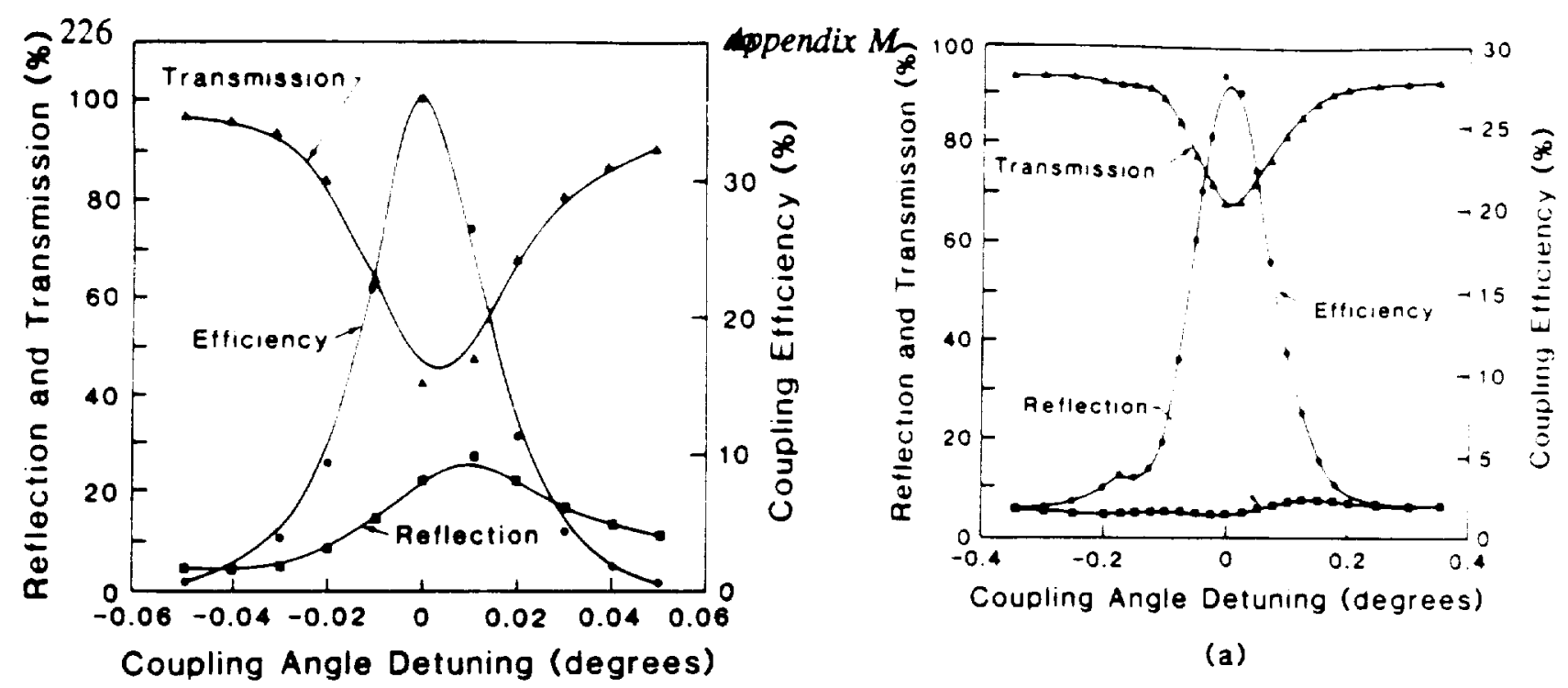

Fin. \&. Expermental data if percentage transmissiun. retlection. and coupling efficiency angular detuning tirr a fiabsian beam of llimims angular width.

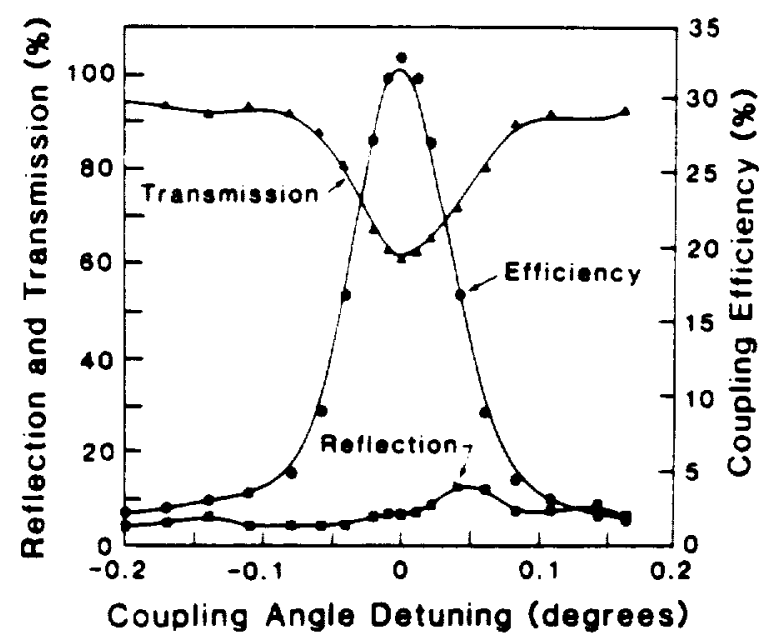

Fig. 5. Experimental data of percentage transmission, reflection. and couplung etficiency 's angular detuning for a Gaussian beam of (i.1). $8^{\circ}$ angular width.

account. However. for a study of the angular dependence of the coupling efficiency, this definition suftices.

The experimental values of the transmission. reflection, and coupling efficiency for a Gaussian beam with a $0.0085^{\circ}$ angular width are shown in Fig. 4. These values indicate an increase in the transmitted signal and a decrease in the reflected signal as the incident angle is detuned. In addition, the angular position between the transmission minimum and the reflection maximum is shifted. The acceptance angle, measured from the efficiency curve, is $0.029^{\circ}$. Although the peak value of the experimental curve is greater than the theoretical value given in Fig. 3 , the acceptance angles measured from both curves agree well.

The experimental data and the theoretical efficiency curves for Gaussian beams with angular widths of

$0.058^{\circ}$ and $0.13^{\circ}$ are shown in Figs. 5 and $6(\mathrm{a})$. respec tively. The shapes of these curves are similar to that of the curve shown in Fig. 4. Note that, although the peak efficiency values are comparable with that in $\mathrm{Fig}$. 4. the apparent acceptance angles have increased to $0.085^{\circ}$ and $0.15^{\circ}$, respectively. There is good agree ment between the experimental and theoretical curves. The variation in the coupling efficiency with coupling angle, as measured with the outcoupled beam from the second grating, is illustrated in Fig. $6(b)$. The measured throughput signal curve agrees well with the measured efficiency curve, but the throughput signal has a much higher signal-to-noise ratio.

The experimental transmission, reflection, and coupling efficiency data for a Gaussian beam with a $0.48^{\circ}$ angular width are shown in Fig. 7 (a). For this case, the 


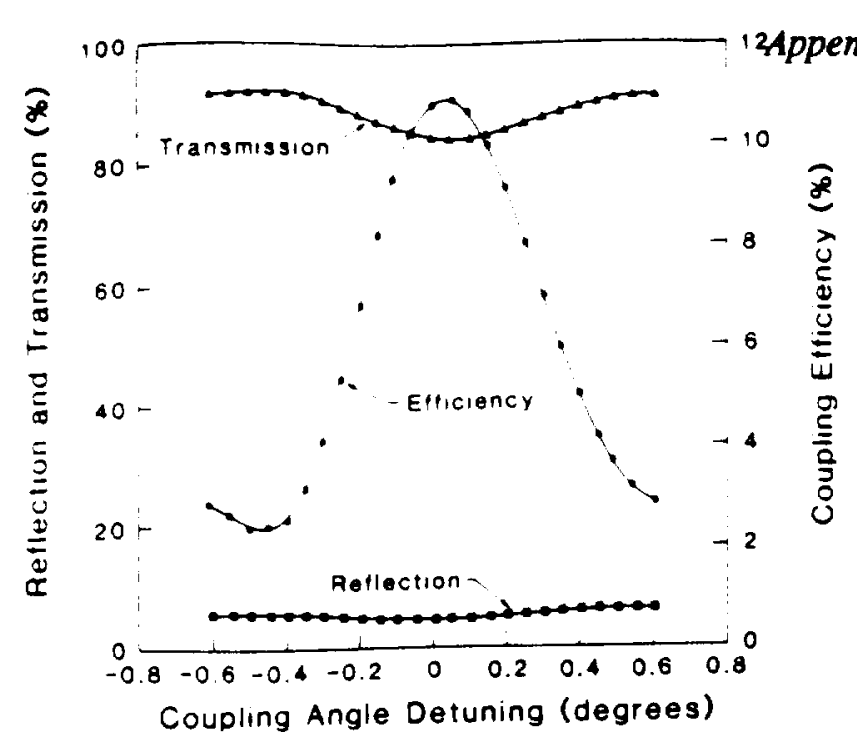

(a)

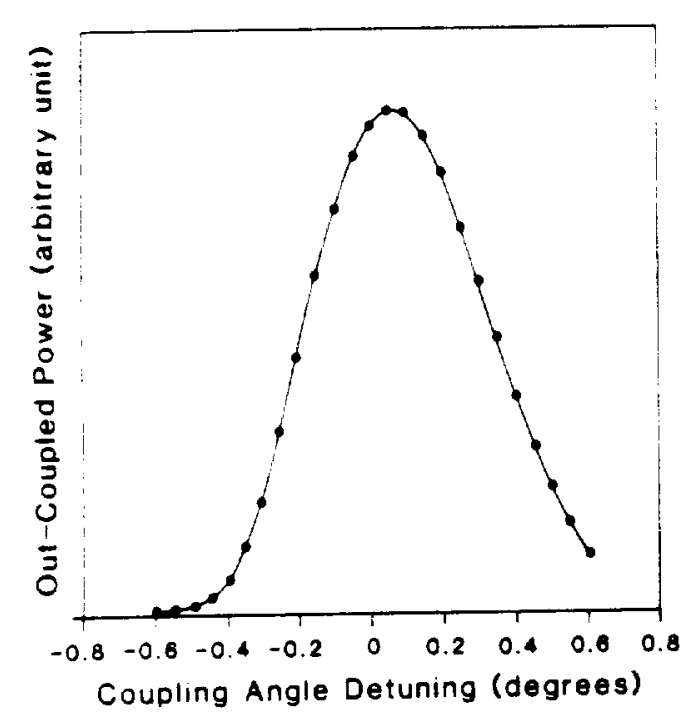

(b)

Fin. - Experimental data of lal percentage transmission, reflecthen. and coupling efficiency. and (b) outcoupled power is angular detuning for a Gaussian beam of $0.48^{\circ}$ angular width.

acceptance angle rises to $0.57^{\circ}$ and the peak efficiency falls to $11 c^{\circ}$. The apparent acceptance angle is about twenty times and the peak efficiency about two-thirds that for the beam of $0.0085^{\circ}$ angular width. The rise in efficiency around $-0.4^{\circ}$ detuning arises from sub. strate mode coupling. A simple calculation shows that. for the grating coupler used in the measurements, the onset coupling angle for the substrate mode is about $-0.4^{\circ}$ from the optimum coupling angle for the guided mode. Thus, as the coupling angle is detuned in the negative direction, a portion of the input beam is coupled into the substrate. Because of the way we define coupling efficiency, this substrate coupling effect appears in the efficiency curve. No such effect is seen in the beam outcoupled by the second grating [see

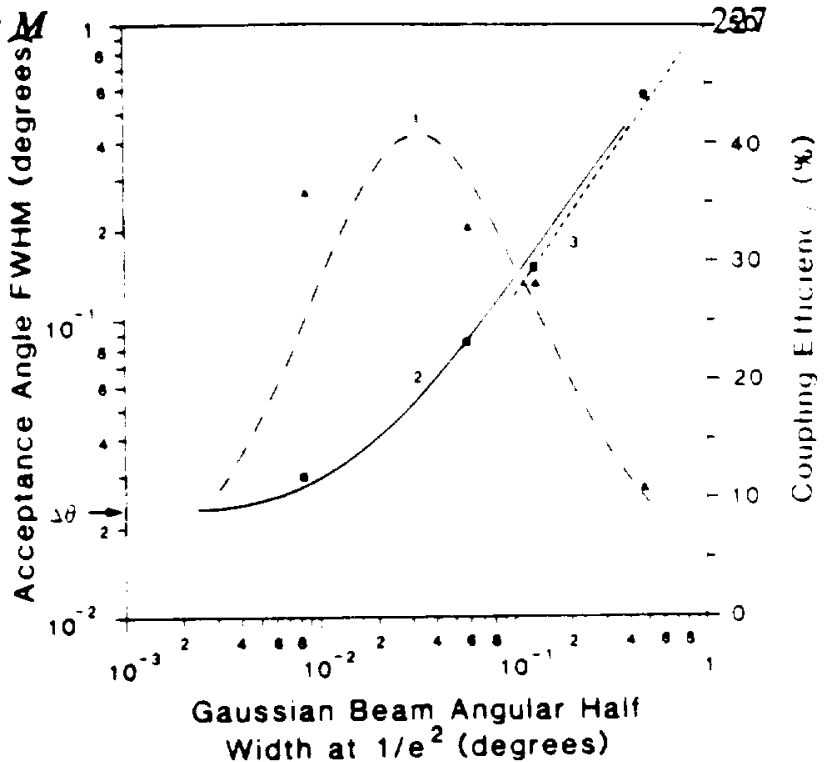

Fig. b. Experimental and theuretical diceptance ans.t $\ldots \ldots$

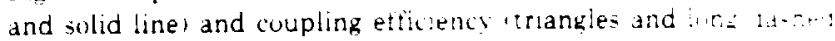
line is Gaussian beam angular width.

Fig. $7($ b) I. Likewise, this effect did not appear in our earlier experiments because the acceptance angles were well under $0.4^{\circ}$. It is not known why the shoulder on the left of the theoretical efficiency curve does not appear on the experimental curve. Despite the difference in the shapes of the experimental and theoretical efficiency curves, the acceptance angles meastired from these two curves are nearly equal.

\section{v. Discussion and Conclusions}

The experimental results and theoretical calculations for the four focused beams are presented in Fig. 8 . The horizontal axis is taken as the angular halfwidth of the incident Gaussian beam at $1 / e^{-2}$. All the curves and data points share this axis. Curve 1 represents the theoretical coupling efficiency and curve 2 the theoret ical apparent acceptance angle; the triangles and squares are the respective experimental points. The arrow indicates the position of the intrinsic acceptance angle $\Delta \theta_{i}$ of the grating coupler on the vertical axis. Curve 3 shows the calculated relationship between the full width at half-maximum and the halfwidth at $1 \mathrm{e}$ maximum for a Gaussian beam. As indicated in Sec. II, as the angular width of the incident beam decreases. the apparent acceptance angle of the grating coupler approaches the intrinsic acceptance angle. Furthermore, as the angular width of the incident beam in creases, the apparent acceptance angle approaches the width of the incident beam. Also, at the maximum coupling efficiency, the apparent acceptance angle is about twice the width of the incident beam. Except for two efficiency data points, the experimental data and the theoretical curves are in good agreement.

Note that, in Fig. 8, the coupling efficiency is plotted on a linear scale, while the acceptance angle is plotted on a logarithmic scale. It can be seen that, for a small 
228 several times by using a more tightly focused beam. Fur example, to achieve the $+2 \mathrm{c}$ maximum coupling efficiencr. une must use a Gaussian beam with a $0.03^{\circ}$ angular halfwidth. tor which the apparent acceptance angle is $-11.126^{\circ}$. If one is content with a $30^{\circ}$ c coupling effictency - 25r less than the maximum valuel and Whes tw druthle the acceptance angle one can do so by: tricusng the incident beam to an angular halfwidth of $11.1^{\circ}$

The asymutic hehavior of curve 2 at the lower end -ungests that the intrinsic acceptance angle can be probed by a sutticiently collimated beam. Because the intrinsic acceptance angle is directly proportional (1) the leakage factor for the grating coupler. one could easily measure the leakage factor.

summarizing. we have conducted a theoretical and experimental study of the angular dependence of the coupling efficiency for a planar waveguide grating coupler. Our results show that. by appropriately focusing the incident Gaussian beam, the acceptance angle of the coupler can be greatly increased with only a small decrease in the maximum coupling efficiency. We also show good agreement between theoretical calculations and experimental measurements. This study allows one to estimate the acceptance angle and coupling efficiency for a grating coupler with a focused incident beam. These findings are useful in applications where the angular tolerance of waveguide coupling is an important issue.

The authors would like to thank T. A. Strasser and G. Wagner of Eastman Kodak's Research Laboratories for their technical assistance. $\mathrm{Li}$ would like to thank the Diversified Technologies Research Laboratories

MTRL of Kodak for providing tinancial support dur. ing his visit there. This research is suppurted bs DTRL of Kodak and the Optical Data Sturage Center at the Lniversity of Arizona.

\section{Relerences}

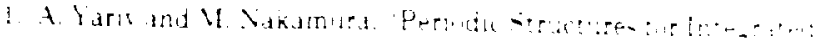

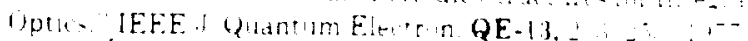

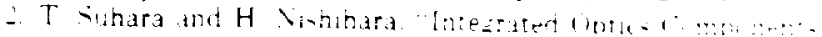

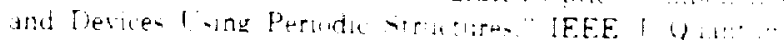

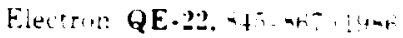

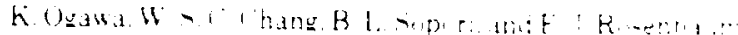

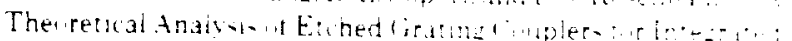

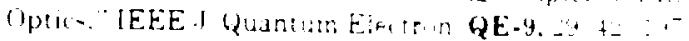

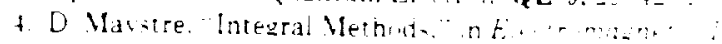

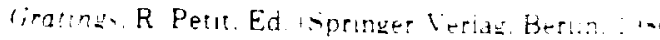
Liil.

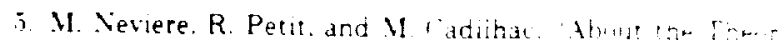
Optical Cratıng Coupler-Wavezuide sistems." opr l l..... 8. 11:-11: 19:31

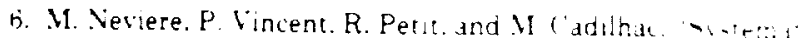

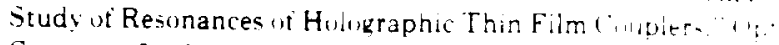
Commun. 9. 48-5.3119-31

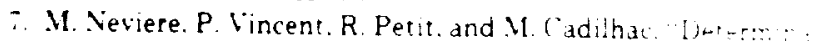

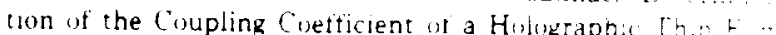
Coupler." Opt. Commun. 9. 2411-24.j,14? :

\&. W Veviere. "The Homogeneous Problem." in E.......... Thetr: w Gratings. R. Petit. Ed Apringer-litiag. Brr 19801. pp. 123-15\%.

9. M. C. Gupta and T. A. Strasser. "Eletron-Beam-Dep .....: Corning 70.59 Glass Thin Films tur Waveguides." Prim - , Phoco-Opt. Instrum. Eng. 993, 4t-ti, 19841.

10. M. C. Gupta. "Low-Optical-Luss Glass Thun Film-" topi lin! 29, 4310-4314+19901.

11. L. Li, M. Xu. G. I. Stegeman. and C. T. Collin Faing $F_{1}$ Photoresist Masks for Submicrometer Surtace Reliel ir a : ? Proc Soc. Photo-Opt. Instrum. Eng. 835, 79-2: 14n- 